Russian Academy of Sciences, Far Eastern Branch

Botanical Garden-Institute

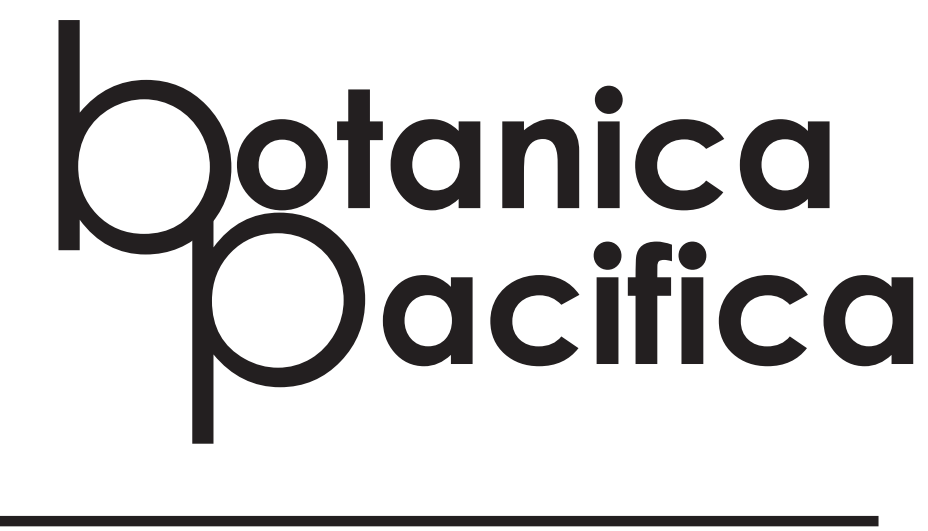

A JOURNAL OF PLANT SCIENCE
AND CONSERVATION

VOLUME 9, NO. 12020 


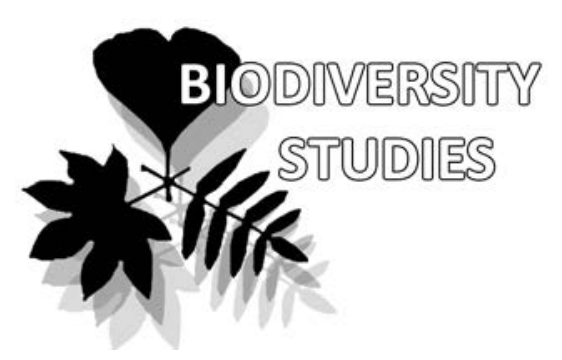

\title{
New data on Myxomycetes of Lazovsky State Nature Reserve (Far East, Russia)
}

\author{
Vladimir I. Gmoshinskiy ${ }^{1 *}$, Fedor M. Bortnikov ${ }^{1}$, \\ Andrey V. Matveev ${ }^{1} \&$ Yuri K. Novozhilov ${ }^{2}$
}

\section{Vladimir I. Gmoshinskiy ${ }^{1 *}$ e-mail: rubisco@list.ru \\ Fedor M. Bortnikov \\ e-mail: f.m.bortnikov@gmail.com \\ Andrey V. Matveev ${ }^{1}$ \\ e-mail: andrmatveev@gmail.com \\ Yuri K. Novozhilov ${ }^{2}$ \\ e-mail: yurinovozhilov@gmail.com}

${ }^{1}$ Lomonosov Moscow State University, Moscow, Russia

${ }^{2}$ V.L. Komarov Botanical Institute RAS, Saint Petersburg, Russia

* corresponding author

Manuscript received: 24.01 .2020

Review completed: 17.03 .2020

Accepted for publication: 24.03 .2020

Published online: 25.03.2020

\begin{abstract}
A B S T R A C T
We summarize all available data on the diversity of myxomycetes of the Lazovsky Nature Reserve and its surroundings, including 494 newly collected specimens. Myxomycete biota of the Lazovsky Nature Reserve consists of 90 species. Fifteen species were added to the checklist of myxomycetes from Primorye Territory. Two rare species, Stemonitis marjana Y. Yamam. and Clastoderma pachypus Nann.-Bremek., are illustrated and discussed.

Keywords: Amoebozoa, biodiversity, checklist, Primorye Territory, Myxomycetes, rare species

\section{P Е 3 Ю М E}

Гмошинский В.И., Бортников Ф.М., Матвеев А.В., Новожимов Ю.К. Новые Аанные о миксомицетах $\Lambda$ азовского государственного заповеАника (Аамьний Восток, Россия). В статье приведены обобщенные данные о биоте миксомицетов Аазовского государственного природного заповеАника имени $\Lambda . Г$. Капланова и его окрестностей, вкАючая информацию о 494 образцах, собранных авторами. Биота миксомицетов заповедника представлена 90 видами, 15 из которых явАяются новыми Аля Приморского края. В работе приведены описания и илАюстрации двух редких видов: Stemonitis marjana Y. Yamam. и Clastoderma pachypus Nann.-Bremek.
\end{abstract}

Ключевые слова: Amoebozoa, биоразнообразие, миксомицеты, Приморский край, редкие виды, список видов

\section{INTRODUCTION}

Myxomycetes are a group of plasmodial slime molds included in Amoebozoa (Adl et al. 2019). Their life cycle combines three stages: mononuclear phase (amoeboid or flagellated), multinuclear phase (plasmodium), and fruiting bodies (sporophores) that contain spores. They live in almost all terrestrial ecosystems and are particularly abundant in temperate and tropical forests (Novozhilov et al. 2017a). Myxomycetes diversity in Russia has been unevenly studied. Over 420 species of myxomycetes are reported for Russia (Matveev et al. 2016-2019). Despite the fact that over 187 species were found in the Far East, this region remains understudied. There was only one regular research on species diversity of myxomycetes (in Sikhote-Alin State Nature Biosphere Reserve) (Novozhilov et al. 2017b). In addition, one local species inventory was compiled in Ussuriysky State Nature Reserve, Kedrovaya Pad' Nature Reserve, and the vicinity of the city of Vladivostok (Bunkina \& Koval 1967, Bunkina \& Nazarova 1978). The only study of myxomycetes in Lazovsky State Nature Reserve was performed by Novozhilov \& Krusanova in 1986. They studied nullah of Benevka (Sandagou) River, where broad-leaved and mixed broad-leaved forests are predominant, and the vicinity of Preobrazheniye urban locality, where oak forests are predominant. In this study, 33 species from 17 genera and 7 families were discovered (Novozhilov \& Krusanova
1989). In 2015, Antonov collected about 60 specimens near Valentine and Lazo settlements and Oblachnaya mountain (Gmoshinskiy \& Antonov 2016). The results of previously studies are included in this checklist.

Lazovsky State Nature Reserve (hereinafter referred to as Lazovsky Nature Reserve) is located on the southeastern slopes of the Sikhote-Alin mountain range, down towards the coast of the Sea of Japan, located within Lazovsky district of Primorye Territory (Russian Far East) between $42^{\circ} 45^{\prime}$ and $43^{\circ} 40^{\prime} \mathrm{N}$ and $133^{\circ} 35^{\prime}$ and $134^{\circ} 15^{\prime} \mathrm{E}$. The reserve occupies an area of $1210 \mathrm{~km}^{2}$, including small Petrov and Beltsov islands ( $1370 \mathrm{~km}^{2}$ including its partly protected buffer zone).

The general features of the climate of the reserve are determined by the proximity of the sea; however, there are significant climatic contrasts on its territory due to the $\mathrm{Za}$ povedny Ridge acting as a barrier to air masses. The winter is cold in northern part of the reserve. The mean temperature in January is from -19 to $-20^{\circ} \mathrm{C}$. Snow normally covers the ground in the end of October or in the first part of November and reaches a depth $50-60 \mathrm{~cm}$. The spring starts in the second part of March. The summer is warm, mean temperature of July and August is $19-20^{\circ} \mathrm{C}$. First frosts are observed from the first half of September. The mean annual precipitation is $700-800 \mathrm{~mm}$. On seaside of reserve the winter is not so cold. The mean temperature of January ranges from -11 to $-12^{\circ} \mathrm{C}$, temperature minimum $-30^{\circ} \mathrm{C}$. In the sea-faced part of 
the reserve, spring starts earlier, but temperature increases not as fast as in the continental part. Cool and humid summers with frequent fogs are determined by the influence of the sea cooling during the winter. The mean August temperature is $16-18^{\circ} \mathrm{C}$; the annual precipitation is $750-850 \mathrm{~mm}$ and can reach $1000-1200 \mathrm{~mm}$ in some years. Typhoons have been observed from the second half of August, when monthly precipitation falls during the day. The soil cover is represented by brown forest soils (Vasilyev et al. 1985).

The reserve is $95 \%$ forested, including the largest yew grove (Taxus cuspidata Siebold et Zucc.) in Russia on Petrov island. The most widespread forests are Quercus mongolica Fisch. ex Turcz. dominated forests, mixed broadleavedKorean pine forests (with Pinus koraiensis Siebold et Zucc.), fir-spruce, birch, and aspen forests. Broadleaved forests are located in the valleys of the rivers. Riparian forests of Chosenia arbutifolia (Pall.) A.K. Skvortsov and species of Salix are situated in the thin band along the river path. Populus maximowiczii A. Henry forests occupy wide areas of valleys. The lowest parts of floodplain, with excessive moistening, are occupied by alder forests. Upper timberline is partially represented by sparse Betula lanata (Regel) V.N. Vassil. trees. Contemporary vegetation of the reserve developed under anthropogenic pressure, primarily burning and timber cutting. As a result, the type of vegetation was changed in the first half of 20th century (Egorova 2002).

\section{MATERIALS AND METHODS}

Field surveys of myxomycetes were performed at various localities of Lazovsky Nature Reserve during August 2015 and June 2016 by I.A. Viner, F.M. Bortnikov, and E.A. Antonov. Georeferences were assigned to all collections using the WGS84 datum and the information, obtained with a Garmin Dakota 20 GPS unit. Specimens of myxomycete sporophores were collected in the field. In the present study, a collection is defined as one or more fruiting bodies considered to have the same plasmodium of origin (Stephenson 1989). Additionally, our checklist also included previously published results from the same territory (see list of collection sites, Fig. 1).

In addition, a few samples of plant litter and bark of living trees from Petrov Island (Loc 1 in list of collection sites) were removed and taken into the laboratory in small paper bags and used to prepare moist chamber cultures according to Stephenson \& Stempen (1994). The moist chambers consisted of glass Petri dishes $(10 \mathrm{~cm}$ diam.) lined with filter paper. Fragments of plant material and sterile water were added into each moist chamber culture. After 12-24 hours, excess water was removed. Cultures were kept at room temperature $\left(18-25^{\circ} \mathrm{C}\right)$ and in ambient daylight. Moist chamber cultures were examined with stereomicroscope each 10 days for up to 3 months or more in order to detect plasmodia and/or fruiting bodies. Myxomycete plasmodia and fruiting bodies were recorded each time the cultures were checked, and all fruiting bodies were removed, air-dried, and fixed in small match-boxes with glue. All sporophores of a given species that developed in the same culture were considered to represent a single record.
All specimens are deposited into the Myxomycetes Collection at the Department Mycology and Algology, Biological faculty of Moscow State University (MYX). The micrographs were made by Leica M 80 dissecting microscope and in transmitted light by Leica D 500. For identification, sporophores were often preserved as temporary slides in $3 \% \mathrm{KOH}$. Some specimens were also examined and photographed with a JEOL JSM-6380LA scanning electron microscope.

We used classification proposed by Lado \& Eliasson (2017) and terms for taxonomic descriptions according to Lado \& Pando (1997) and Poulain et al. (2011a).

\section{List of collection sites}

\section{Collection sites studied in 2016}

1: Petrov Island, grove of Taxus cuspidata, $42.86578^{\circ} \mathrm{N}$ $133.80510^{\circ} \mathrm{E}(21.06 ; 29.06 ; 07.07)$.

2-3: Petrov Island. Mixed forest. $2: 42.86345^{\circ} \mathrm{N} 133.80544^{\circ} \mathrm{E}$ (21.06; 07.07); 3: $42.86572^{\circ} \mathrm{N} 133.80802^{\circ} \mathrm{E}(04.07)$.

4; 12; 23-34: Mongolian oak forest (Quercus mongolica). 4: $42.87667^{\circ} \mathrm{N} \quad 133.78458^{\circ} \mathrm{E} \quad(09.07) ; 12: 42.87683^{\circ} \mathrm{N}$ $133.79358^{\circ} \mathrm{E}(29.06) ; 23: 42.87461^{\circ} \mathrm{N} 133.79922^{\circ} \mathrm{E}(15.06)$; 24: $42.87767^{\circ} \mathrm{N} 133.79908^{\circ} \mathrm{E}(21.06) ; 25: 42.87683^{\circ} \mathrm{N}$ $133.80003^{\circ} \mathrm{E}(04.07) ; 26: 42.87575^{\circ} \mathrm{N} 133.80450^{\circ} \mathrm{E}(02.07)$; 27: $42.87725^{\circ} \mathrm{N} 133.80628^{\circ} \mathrm{E}(28.06) ; 28: 42.87939^{\circ} \mathrm{N}$ $133.80514^{\circ} \mathrm{E}(30.06) ; 29: 42.87978^{\circ} \mathrm{N} 133.80269^{\circ} \mathrm{E}(02.07)$; 30: $42.89375^{\circ} \mathrm{N} 133.82644^{\circ} \mathrm{E}$ (13.06); 31: $42.89519^{\circ} \mathrm{N}$ 133.82753 ${ }^{\circ} \mathrm{E}(20.06) ; 32: 42.89408^{\circ} \mathrm{N} 133.83003^{\circ} \mathrm{E}(13.06)$; 33: $42.89267^{\circ} \mathrm{N} 133.83017^{\circ} \mathrm{E}$ (20.06); 34: $42.89892^{\circ} \mathrm{N}$ $133.84883^{\circ} \mathrm{E}(13.06)$.

5-11; 13; 18-22; 35-40: forest with Alnus, Populus, Fraxinus, Betula, Carpinus, and Acer. 5: $42.87533^{\circ} \mathrm{N} 133.78492^{\circ} \mathrm{E}$ (07.06); 6: $42.87636^{\circ} \mathrm{N} 133.78911^{\circ} \mathrm{E}(06.07) ; 7: 42.87681^{\circ} \mathrm{N}$ $133.79089^{\circ} \mathrm{E}(06.07) ; 8: 42.87742^{\circ} \mathrm{N} 133.79100^{\circ} \mathrm{E}(06.06)$; 9: $42.87744^{\circ} \mathrm{N} 133.79142^{\circ} \mathrm{E} \quad(06.06) ; 10: 42.87756^{\circ} \mathrm{N}$ $133.79250^{\circ} \mathrm{E}(22.06) ; 11: 42.87656^{\circ} \mathrm{N} 133.79244^{\circ} \mathrm{E}(22.06)$; 13: $42.87597^{\circ} \mathrm{N} \quad 133.79442^{\circ} \mathrm{E} \quad(07.06) ; 18: 42.87278^{\circ} \mathrm{N}$ $133.79317^{\circ} \mathrm{E}(17.06) ; 19: 42.87311^{\circ} \mathrm{N} 133.79353^{\circ} \mathrm{E}(17.06)$; 20: $42.87356^{\circ} \mathrm{N} 133.79553^{\circ} \mathrm{E}(15.06) ; 21: 42.87447^{\circ} \mathrm{N}$ $133.79561^{\circ} \mathrm{E}(17.06) ; 22: 42.87569^{\circ} \mathrm{N} 133.79644^{\circ} \mathrm{E}(07.06$; 18.06; 19.06); 35: $42.89906^{\circ} \mathrm{N} \quad 133.85042^{\circ} \mathrm{E} \quad(14.06)$; 36: $42.89931^{\circ} \mathrm{N} 133.85208^{\circ} \mathrm{E}(10.07) ; 37: 42.89911^{\circ} \mathrm{N}$ 133.85531 ${ }^{\circ} \mathrm{E}(14.06) ; 38: 42.89986^{\circ} \mathrm{N} 133.85617^{\circ} \mathrm{E}(14.06)$; 39: $42.90072^{\circ} \mathrm{N} 133.85844^{\circ} \mathrm{E}(13.06) ; 40: 42.90369^{\circ} \mathrm{N}$ $133.86025^{\circ} \mathrm{E}(14.06)$.

14: wood house, $42.87120^{\circ} \mathrm{N} 133.79318^{\circ} \mathrm{E}(07.06)$.

15: waterlogged area near ambit of cordon, $42.87169^{\circ} \mathrm{N}$ $133.79316^{\circ} \mathrm{E}(05.06 ; 13.06)$.

16-17: meadow at the cordon, $16: 42.87151^{\circ} \mathrm{N} 133.79444^{\circ} \mathrm{E}$ (05.06); 17: $42.87245^{\circ} \mathrm{N} 133.79447^{\circ} \mathrm{E}$ (12.06).

\section{List of collection sites studied in 2015}

(Gmoshinskiy \& Antonov 2015).

41-42, 53, 55: Near Valentin locality. 41: $43.12527^{\circ} \mathrm{N}$ 134.28922 ${ }^{\circ} \mathrm{E}(13.08) ; 42: 43.12537^{\circ} \mathrm{N} 134.28932^{\circ} \mathrm{E}(13.08)$; 53: $43.124122^{\circ} \mathrm{N} 134.315077^{\circ} \mathrm{E}(13.08) ; 55: 43.126717^{\circ} \mathrm{N}$ $134.29173^{\circ} \mathrm{E}(13.08)$.

43-46, 56: Near Lazo locality. 43: $43.35797^{\circ} \mathrm{N} 133.90797^{\circ} \mathrm{E}$ (15.08); $44: 43.36683^{\circ} \mathrm{N} 133.90677^{\circ} \mathrm{E}(15.08) ; 45: 43.37510^{\circ} \mathrm{N}$ $133.90822^{\circ} \mathrm{E}(15.08) ; 46: 43.37533^{\circ} \mathrm{N} 133.90897^{\circ} \mathrm{E}(15.08)$; 56: $43.372287^{\circ} \mathrm{N} 133.896738^{\circ} \mathrm{E}(15.08)$.

47-53: Oblachnaya Mountain. 47: 43.65836 ${ }^{\circ} \mathrm{N} 134.29753^{\circ} \mathrm{E}$ (17.08); 48: $43.68118^{\circ} \mathrm{N} 134.20181^{\circ} \mathrm{E}(17.08) ; 49: 43.68341^{\circ} \mathrm{N}$ 
134.20018 ${ }^{\circ} \mathrm{E}(17.08) ; \mathbf{5 0 :} 43.69190^{\circ} \mathrm{N} 134.19630^{\circ} \mathrm{E}(17.08) ;$ 51: $43.69808^{\circ} \mathrm{N} \quad 134.20029^{\circ} \mathrm{E} \quad(17.08) ; \quad 52: 43.69950^{\circ} \mathrm{N}$ $134.20142^{\circ} \mathrm{E}(17.08)$.

54: South spur of Snezhnaya Mountain, $43.725587^{\circ} \mathrm{N}$ $134.43771^{\circ} \mathrm{E}(18.08)$.

List of collection sites, studied in 1986

(Novozhilov \& Krusanova 1989).

L1-L3: Cordon Sandagou, Benevka river. L1: floodplain broad-leaved forest (01.10; 03.10); L2: pine-broad-leaved forest (29.09; 01-03.10; 08.10); L3: broad-lived forest (29.09; 01.10; 3.10).

L4: Near Preobrazheniye urban locality, oak forest (05.10; 09.10).

\section{RESULTS}

The following annotated checklist was compiled from our quantitative survey of 456 field specimens and 38 collections from moist chambers and literature data (Novozhilov \& Krusanova 1989). The abbreviations for these data are given in Table 1.

†Arcyria affinis Rostaf., FC: 4: 4 (MYX 7153); 6: 4 (MYX 7193); 7: 2 (MYX 7209); 36: 1; 46: 1; 56: 1 (MYX 6367).

†Arcyria cinerea (Bull.) Pers., FC: 4: 2 (MYX 7152); 6: 4 (MYX 7192); 7: 3 (MYX 7215); 10: 1 (MYX 7238); 11: 1 (MYX 7251); 25: 7 (MYX 7324); 36: 2; 42: 1 (MYX 6368); 43: 1 (MYX 6370); 45: 1; 46: 2 (MYX 6365); 54: 2 (MYX 6382). MC: 1: 5 (MYX 8146, MYX 8147, MYX 8148, MYX 8149, MYX 8172).

†Arcyria denudata (L.) Wettst., FC: 6: 1 (MYX 7191); 27: 1; 36: 2; 41: 1 (MYX 6377).

†Arcyria ferruginea Saut., FC: 22: 1.

Arcyria incarnata (Pers. ex J.F. Gmel.) Pers., FC: 6: 1; 10: 1 (MYX 7239); 25: 2 (MYX 7321, MYX 7325); L1

*Arcyria cf. magna Rex., FC: 1: 1 (MYX 7131); 22: 1 .

*Arcyria oerstedii Rostaf., FC: 1: 1 (MYX 7130). †Arcyria pomiformis (Leers) Rostaf., FC: 4: 1 (MYX 7150); 7: 2 (MYX 7216); 25: 1; 50: 1. MC: 1: 2 (MYX 8166, MYX 8173).

Arcyria stipata (Schwein.) Lister, FC: L1

*Badhamia affinis Rostaf., FC: 7: 1 (MYX 7227); 24: 1 (MYX 7310).
* Badhamia capsulifera (Bull.) Berk., FC: 18: 1 (MYX 7274). Figs 3A, B.

†Badhamia foliicola Lister., FC: 20: 1 (MYX 7280).

†Badhamia utricularis (Bull.) Berk., FC: 23: 3 (MYX 7305, MYX 7306, MYX 7307). All specimens were found on fruiting bodies of polyporoid fungi.

†Calomyxa metallica (Berk.) Nieuwl., MC: 1: 1 (MYX 8174). †Ceratiomyxa fruticulosa var. flexuosa (Lister) G. Lister, FC: 1: 1 (MYX 7135); 3: 1 (MYX 7141); 4: 3 (MYX 7148); 6: 4 (MYX 7184); 7: 5 (MYX 7206); 8: 1 (MYX 7230); 10: 6 (MYX 7234); 12: 2 (MYX 7259); 22: 1 (MYX 7287); 25: 2; 26: 2 ; 28: 2; 35: 1; 36: 1

†Ceratiomyxa fruticulosa var. porioides (Alb et Schwein.) G. Lister, FC: 1: 1 (MYX 7132); 6: 1 (MYX 7185); 7: 4 (MYX 7205, MYX 7328); 10: 1 (MYX 7232); 11: 2 (MYX 7249); 12: 1 (MYX 7258).

Clastoderma debaryanum A. Blytt., FC: L1; L2; L4. MC: 1: 1 (MYX 8175).

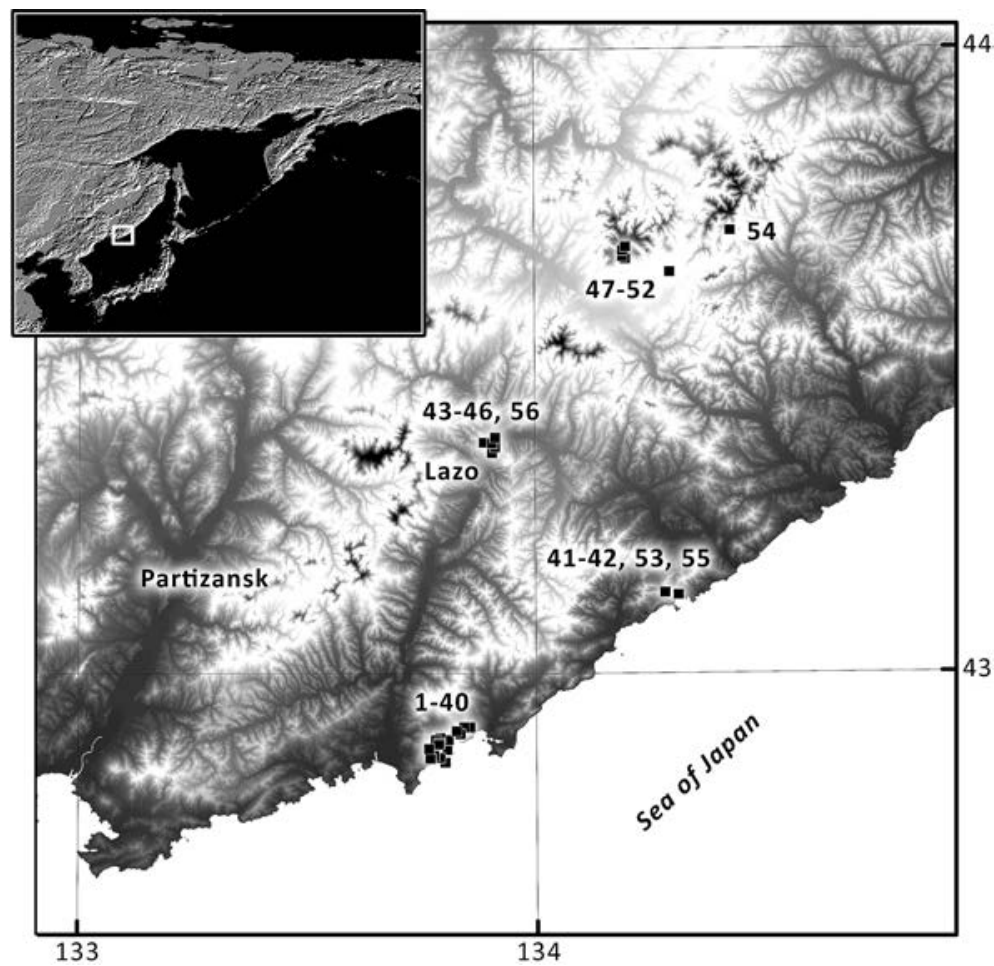

Figure 1 Schematic map of the studied area in Lazovsky State Nature Reserve and its vicinity in 2015-2016

Table 1. Abbreviations used in the annotated species list

\begin{tabular}{|c|c|c|}
\hline Example & Data & Description \\
\hline$\dagger{ }^{*}$ & Status & $\begin{array}{l}\text { The species marked with } \uparrow \text { or } * \text { are new records for Lazovsky Nature Reserve and Primorye } \\
\text { Territory, respectively }\end{array}$ \\
\hline Arcyria affinis & Taxon name & \\
\hline Rostaf. & Author & According to Lado (2005-2019), unless stated otherwise \\
\hline $\mathrm{FC}, \mathrm{MC}$ & Sampling method & FC - field sampling; $\mathrm{MC}$ - moist chambers \\
\hline 4 & Collection site & List of collection sites where the species was found (see list of collection sites and Fig. 1) \\
\hline 4 & Record number & Number of records from this locality \\
\hline (MYX 7153) & Specimen & $\begin{array}{l}\text { Specimens from Collection of Myxomycetes at the Department of Mycology and Algology } \\
\text { (Faculty of Biology, Lomonosov Moscow State University) (MYX). The absence of a herbarium } \\
\text { number in the list means that the species was only registered and the specimen has not yet been } \\
\text { placed in the herbarium }\end{array}$ \\
\hline
\end{tabular}



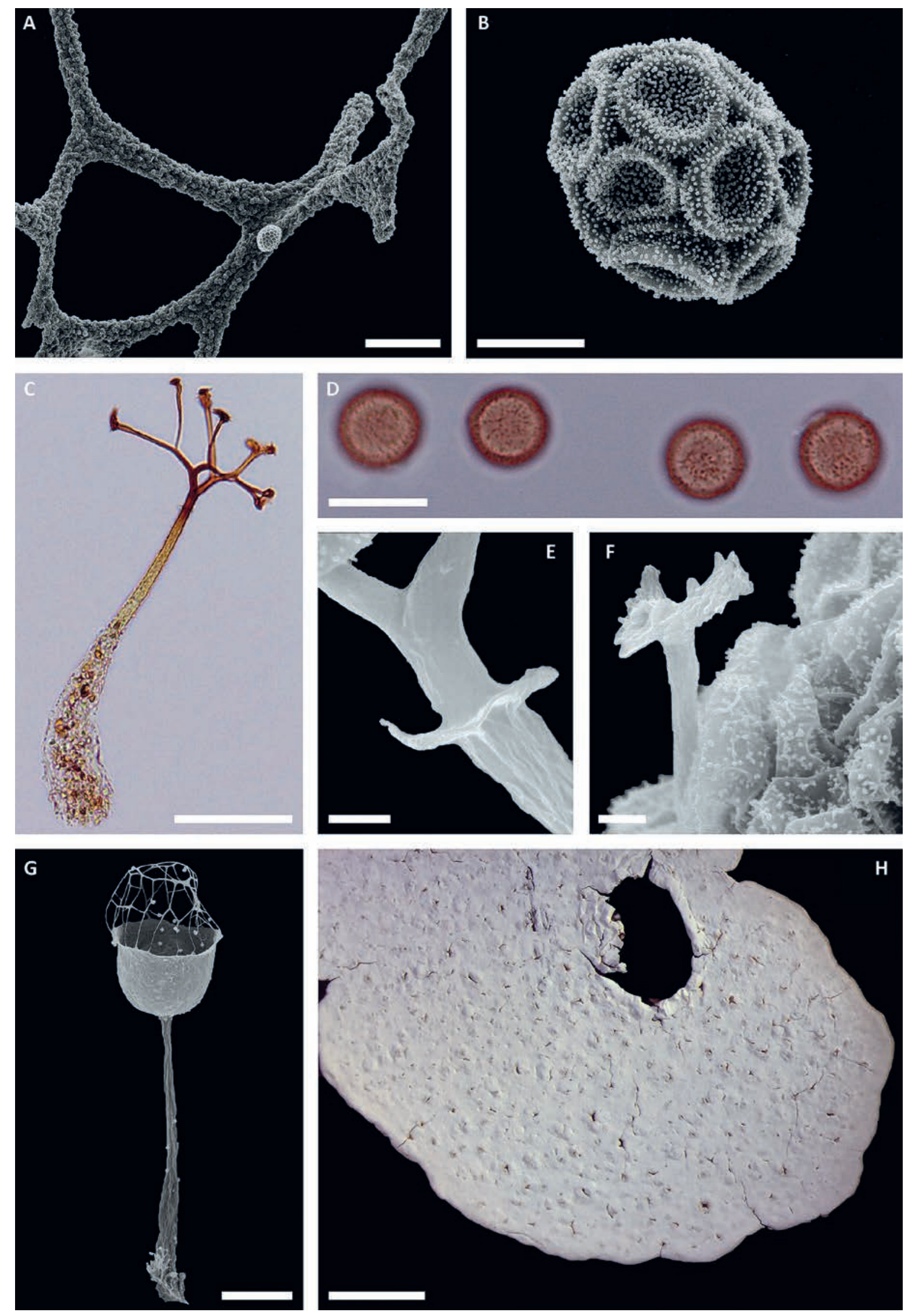

Figure 2 Badhamia capsulifera (Bull.) Berk. (A, B): A - capillitium as seen by scanning electron microscope (SEM) with single spore of Tubifera sp., B - clustered spores (SEM); Clastoderma pachypus Nann.-Bremek. (C-F): C - sporocarp seen with light microscopy (LM), $\mathrm{D}$ - spores (LM), E - collar at the base of sporocyst (SEM), F - plate-like widening at the end of capillitium thread and spores (SEM). G - Cribraria minutissima Schwein., sporocarp (SEM). H - Diderma saundersii (Berk. et Broome ex Massee) E. Sheld., plasmodiocarp (dissecting microscope, DM). Bars: $A=20 \mu \mathrm{m}, \mathrm{B}=10 \mu \mathrm{m}, \mathrm{C}=50 \mu \mathrm{m}, \mathrm{D}=15 \mu \mathrm{m}, \mathrm{E}, \mathrm{F}=3 \mu \mathrm{m}, \mathrm{G}=100 \mu \mathrm{m}, \mathrm{H}=2 \mathrm{~mm}$ 

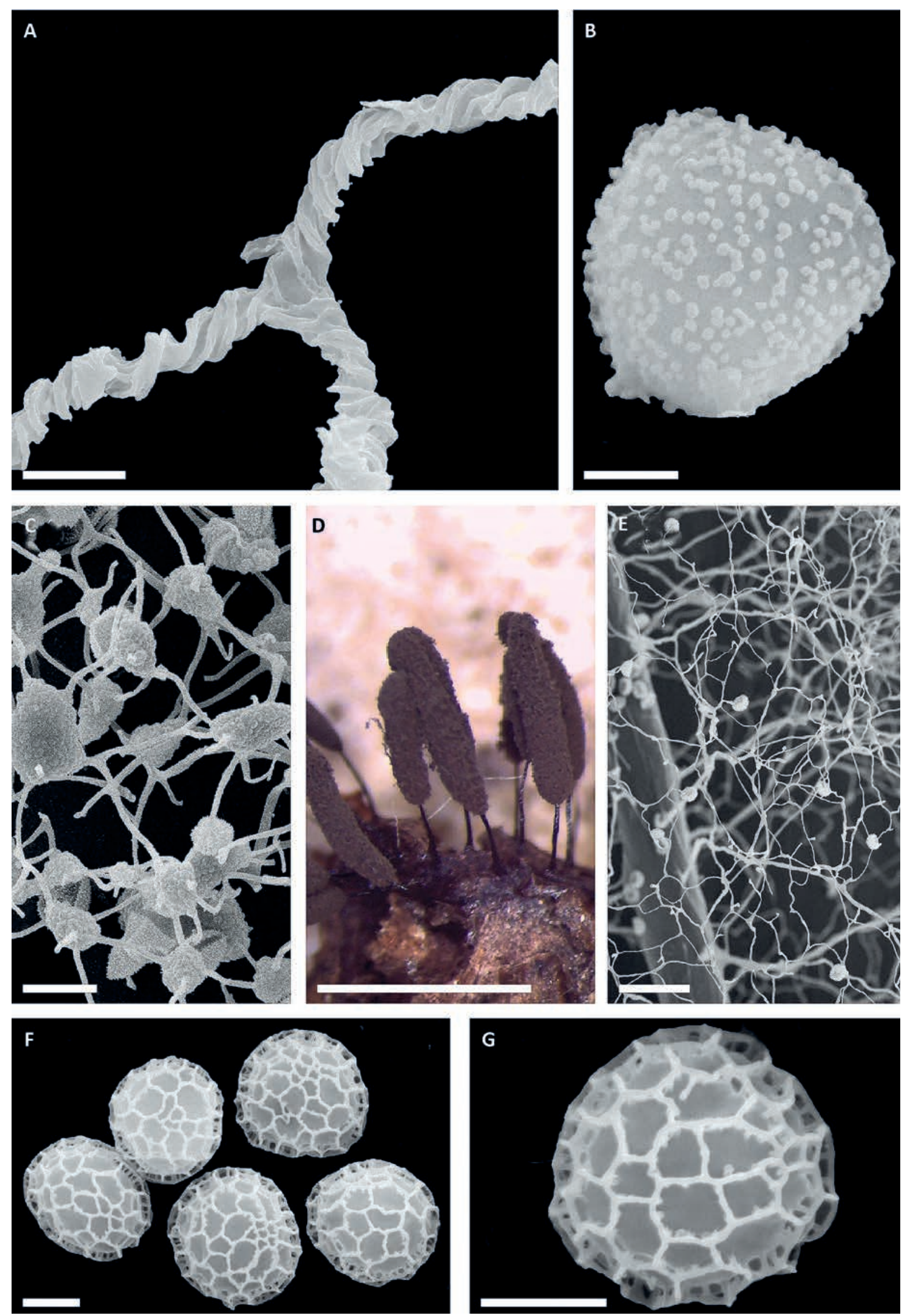

Figure 3 Hemitricbia abietina (Wigand) G. Lister (A, B): A - capillitium as seen by SEM, B - spore (SEM); C - Physarum tenerum Rex., capillitium (SEM); Stemonitis marjana Y. Yamam. (D-G): D - group of sporocarps (dissecting microscope), E - surface net of capillitium (SEM), E, F- spores (SEM). Bars: A, C $=10 \mu \mathrm{m}, \mathrm{B}, \mathrm{F}, \mathrm{G}=3 \mu \mathrm{m}, \mathrm{D}=2 \mathrm{~mm}, \mathrm{E}=30 \mu \mathrm{m}$ 
*Clastoderma pachypus Nann.-Bremek., MC: 1: 1 (MYX 8176). Figs 2C-F.

Sporophores are stalked sporangia, scattered, darkbrown when moist and light-brown when dry, 0.15 $0.3 \mathrm{~mm}$ high, sporangium up to $0.1 \mathrm{~mm}$ in diam. Peridium partly remains as a collar at the base after the upper portion has broken away. Hypothallus is absent or inconspicuous. Stalk long, occupying three-quarters of the total height of the sporophore, expanded at the base, translucent lightbrown, contain refuse matter, tapering in upper part, in upper quarter is brown, translucent, lack granular material. Columella short, is a prolongation of the stipe inside the sporangia, divided dichotomously, evolve into capillitium. Capillitium loose, presented by individual threads, divided dichotomously, with plate-like widenings at the end, do not form close net. Spores in mass brown; light-violaceous in transmitted light, globose, uniformly thickened, 12.5-13.5 $\mu \mathrm{m}$ in diam., very finely and regularly warted. Plasmodium hyaline or pale-brown.

Substrate: on bark of living Taxus cuspidata.

Comparisons with other species with similar morphology of sporophores has been made. C. pachypus has large spores, brown spore mass and stalk without the droplike swelling. If closed net of capillitium is formed, then it is very similar to Echinostelium cribrarioides Alexop., but the latter has vellow spore mass. Clastoderma microcarpum (Meyl.) Kowalski differs by large size of sporophores (0.34-0.62 $\mathrm{mm}$ high and $0.17-0.25 \mathrm{~mm}$ in diam.), black stalk base, more compact capillitium without free ends and expanded plates, and large spores $(13.5-15 \mu \mathrm{m}$ in diam.) (Kowalski 1975).

It is a rare species for Asian region and was previously reported only from East coast of China (Liu et al. 2013) and from eastern Tian-Shan (Schnittler et al. 2013). Our specimen consists of 4-6 mature sporocarps with dispersed spores. It has wide ends of capillitium threads and do not form a close net. Original differential diagnosis states that main differences between C. pachypus and C. debaryanum are small size of sporangium, firm capillitium, and large spores (Nannenga-Bremekamp 1968). The original description states: "Capillitium lax, violet-brown, consisting of a few, rather rigid, not anastomosing branches; the latter ramifying dichotomously, with the ramifications spreading at an almost right angle and ending at the periphery in the persistent flakes of the peridium". Therefore, in the original description and illustration (Nannenga-Bremekamp 1968, fig. 3) there is no mention of a closed network of capillitium. However, later papers provide descriptions and illustrations of specimens with closed large-meshed net of capillitium on the periphery of sporangium (Mitchel 1978: fig. 28; Lado 1985: figs 3-5; Ing 1999: fig. 4; Poulain et al. 2011b: pl. 80). In addition, in some monographs, one of the main identifying features of this species is the presence of a closed peripheral network (Ing 1999, Poulain 2011a). Interestingly, in some cases, the C. pachypus may have a partially closed capillitium, with a small number of free expanded, plate-like ends, and a practically closed peripheral net (Leontyev et al. 2011: fig. 2). Moreover, the specimens of C. pachypus from the hyperarid regions of China (Schnittler et al. 2013) have small spores (7.2-8.0 $\mu \mathrm{m}$ in diam.). Thus, to establish the degree of significance of these morphological traits, a more detailed study of a series of specimens of this rather rare species is required.

Collaria arcyrionema (Rostaf.) Nann.-Bremek. ex Lado, FC: L3.

*Comatricha ellae Härk., FC: 22: 1; $24: 1$ (MYX 7308). †Comatricha laxa Rostaf., FC: 10: 1; 24: 1. MC: 1: 1 (MYX 8167).

†Comatricha nigra (Pers. ex J.F. Gmel.) J. Schröt., in Cohn., FC: 1: 1 (MYX 7134); 4: 1; 6: 1 (MYX 7198); 7: 2 (MYX 7222); 10: 5; 11: 3 (MYX 7252); 12: 3 (MYX 7262); 15: 1 (MYX 7266); 20: 3 (MYX 7276); 22: 3 (MYX 7289); 25: 3 (MYX 7317); 27: 3; 28: 1; 31: 3; 34: 1; 44: 1.
*Comatricha tenerrima (M.A. Curtis) G. Lister, in Lister., FC: 28: 1.

Craterium leucocephalum (Pers. ex J.F. Gmel.) Ditmar, in Sturm, FC: L3.

Craterium obovatum Peck, FC: L2.

Cribraria cancellata (Batsch) Nann.-Bremek., FC: 4: 1 (MYX 7145); 7: 1 (MYX 7207); 10: 2 (MYX 7235); 41: 1 (MYX 6375); L2.

†Cribraria microcarpa (Schrad.) Pers., FC: 4: 3 (MYX 7146); 7: 1 (MYX 7214); 10: 1 (MYX 7231); 25: 6 (MYX 7327); 41: 1 (MYX 6373); 46: 1.

Cribraria minutissima Schwein., FC: 44: 1; L2. Fig. 2G. †Cribraria tenella Schrad., FC: 41: 1 (MYX 6378); 44: 1. †Cribraria violacea Rex., MC: 1: 1 (MYX 8150).

Diachea leucopodia (Bull.) Rostaf., FC: L3.

Diachea splendens Peck, FC: L2.

†Diderma effusum (Schwein.) Morgan., MC: 1: 2 (MYX 8168, MYX 8177).

Diderma montanum (Meyl.) Meyl., FC: L2.

Diderma radiatum (L.) Morgan, FC: L1.

* Diderma saundersii (Berk. et Broome ex Massee) E. Sheld., MC: 1: 1 (MYX 8169). Fig. 2H.

†Didymium anellus Morgan., MC: 1: 1 (MYX 8151).

†Didymium squamulosum (Alb. et Schwein.) Fr. et Palmquist., MC: 1: 1 (MYX 8152).

†Echinostelium minutum de Bary, in Rostafinski., MC: 1: 1 (MYX 8178).

†Enerthenema papillatum (Pers.) Rostaf., FC: 7: 2 (MYX 7213). MC: 1: 1 (MYX 8170).

†Hemitrichia abietina (Wigand) G. Lister, in Lister. MC: 1: 1 (MYX 8171). Figs 3A, B.

Hemitrichia clavata (Pers.) Rostaf., FC: L1.

†Hemitrichia calyculata (Speg.) M.L. Farr., FC: 4: 4 (MYX 7155); 6: 7 (MYX 7194); 7: 8 (MYX 7219); 20: 2 (MYX 7277); 22: 3 (MYX 7288); 25: 4 (MYX 7323); 26: 2; 28: 2; 29: 1; 31: 1; 36: 7; 39: 1; 40: 1; 41: 2 (MYX 6372, MYX 6376); 45: 2; 53: 1 .

†Hemitrichia minor G. Lister., MC: 1: 2 (MYX 8179).

Hemitrichia serpula (Scop.) Rostaf. ex Lister., FC: 4: 2 (MYX 7154); 10: 2 (MYX 7241); 12: 1 (MYX 7260); 29: 1; 30: 1; 37: 2; L4.

Lamproderma arcyrioides (Sommerf.) Rostaf., FC: L2.

Lamproderma columbinum (Pers.) Rostaf., FC: L2.

Lamproderma scintillans (Berk. et Broome) Morgan., FC: L2. MC: 1: 6 (MYX 8153, MYX 8154, MYX 8155, MYX 8156, MYX 8157, MYX 8158).

†Licea operculata (Wingate) G.W. Martin., MC: 1: 1 (MYX 8180).

†Licea parasitica (Zukal) G.W. Martin., MC: 1: 1 (MYX 8181).

†Licea pusilla Schrad., MC: 1: 1 (MYX 8182).

Lycogala epidendrum (L.) Fr., FC: 1: 2 (MYX 7138); 3: 1 (MYX 7143); 4: 1 (MYX 7147); 5: 1 (MYX 7183); 6: 1 (MYX 7187); 7: 1 (MYX 7212); 10: 4 (MYX 7233, MYX 7243); 11: 6 (MYX 7256); 17: 1 (MYX 7268); 18: 1 (MYX 7273); 19: 1 (MYX 7271); 20: 2 (MYX 7275); 21: 1 (MYX 7281); 22: 5 (7285); 25: 2 (MYX 7322); 26: 1; 29: 1; 32: 1; 33: 1; 35: 2; 36: 1; 38: 1; 48: 1; 49: 1; L2.

Lycogala exiguum Morgan., FC: 6: 1 (MYX 7190); 10: 1 (MYX 7236); 44: 1; 46: 1 (MYX 6364); L1.

Metatrichia vesparia (Batsch) Nann.-Bremek. ex G.W. Martin et Alexop., FC: 4: 1 (MYX 7151); 12: 1 (MYX 7261); 
17: 1 (MYX 7269); 20: 1 (MYX 7278); 22: 1 (MYX 7283); 31: 1; 45: 1; L4.

†Perichaena chrysosperma (Curr.) Lister., FC: 24: 1 (MYX 7311). MC: 1: 4 (MYX 8159, MYX 8160, MYX 8161, MYX 8162).

†Perichaena corticalis (Batsch) Rostaf., FC: 11: 1 (MYX 7250).

Physarum album (Bull.) Chevall., FC: 4: 1 (MYX 7163); 6: 2 (MYX 7201); 7: 6 (MYX 7225); 11: 1 (MYX 7257); 44: 1; L2.

†Physarum cinereum (Batsch) Pers., FC: 4: 1 (MYX 7162); 25: 6 (MYX 7313, MYX 7315).

Physarum flavicomum Berk., FC: L2.

†Physarum globuliferum (Bull.) Pers., FC: 55: 1 (MYX 6381).

Physarum leucophaeum Fr. et Palmquist., FC: L2. MC: 1: 1 (MYX 8164).

*Physarum leucopus Link., FC: 1: 1 (MYX 7133).

†Physarum melleum (Berk. et Broome) Massee., MC: 1: 1 (MYX 8163).

Physarum schroeteri Rostaf., FC: L2.

Physarum sulphureum Alb. et Schwein., FC: L1.

†Physarum tenerum Rex., FC: 7: 2 (MYX 7202, MYX 7203). Fig. 3C.

Physarum viride (Bull.) Pers., FC: 4: 1 (MYX 7164); 7: 4 (MYX 7204, MYX 7226); 22: 1 (MYX 7287); 25: 3 (MYX 7314); 36: 2; 44: 1; 45: 1; 55: 1 (MYX 6380); L2.

†Physarum viride var. aurantium (Bull.) Lister, FC: 10: 1 (MYX 7248).

†Physarum viride var. incanum Lister, FC: 14: 1 (MYX 7264).

* Reticularia intermedia Nann.-Bremek., FC: 6: 1 (MYX 7188); 16: 1 (MYX 7270); 24: 1 (MYX 7309); 39: 1.

†Reticularia lycoperdon Bull., FC: 6: 1 (MYX 7189); 8: 1 (MYX 7228); 9: 2 (MYX 7229); 11: 1 (MYX 7253); 15: 1 (MYX 7265); 22: 3 (MYX 7290); 31: 1.

Reticularia splendens Morgan, FC: L2; L4.

Stemonaria irregularis (Rex) Nann.-Bremek., R. Sharma et Y. Yamam. FC: L2.

†Stemonitis axifera (Bull.) T. Macbr., FC: 1: 2 (MYX 7137); 2: 2 (MYX 7140); 3: 1 (MYX 7144); 4: 6 (MYX 7156); 6: 7 (MYX 7196); 7: 9 (MYX 7220); 10: 8 (MYX 7246); 11: 1 (MYX 7254); 25: 1 (MYX 7319); 29: 1; 36: 4; 42: 1; 44: 1; 46: 1 (MYX 6363); 52: 2.

†Stemonitis flavogenita E. Jahn., FC: 2: 1 (MYX 7139); 10: 2 (MYX 7247).

†Stemonitis fusca Roth., FC: 4: 1 (MYX 7161).

* Stemonitis lignicola Nann.-Bremek. (in some publications considered as synonym $S$. splendens, according to Lado 2005-2019). FC: 4: 1 (MYX 7158); 6: 1 (MYX 7199); 7: 2 (MYX 7224); 36: 2.

*Stemonitis marjana Y. Yamam., MC: 1: 1 (MYX 8165). Figs 3D-G.

Sporophores stalked, united in small colony, almost erect, 2.1-2.9 $\mathrm{mm}$ high. Sporangium cylindrical, rounded at base and apex, from red-brown to dark-brown, 0.18$0.25 \mathrm{~mm}$ diam. and 1.6-2.2 mm high. Stalk shiny, slightly tapering upwards, almost black, opaque, $0.5-0.7 \mathrm{~mm}$ high (2/10-3/10 of total height of sporophore). Hypothallus membranous, silvery or light-brown, shared by the whole colony. Peridium completely disappearing, leaving a small collar at the base. Columella almost reaching apex of sporangium, gradually tapering, dark-brown or almost black. Capillitium separate from the columella throughout its length, uniformly thickened, smooth, sometimes with small membranous expansions in junctions, light-brown in transmitted light. Inner net forked and anastomosed 2-4 times, reached periphery of sporangium. Surface net with irregular meshes (5-) 20-50 (-75) $\mu \mathrm{m}$ in diam., with short, pointed free ends. Spores light to dark-brown in mass; dun in transmitted light, globose, with uniformly thick wall, ornamented irregular small-meshed net, with rows of spines or ridges perforated laterally (SEM), 8.5$9.8 \mu \mathrm{m}$ in diam. (including ornamentation $(0.7-0.8 \mu \mathrm{m}$ in height). Plasmodium unknown.

It is the second finding of this species in Russia. It was also recorded from Sverdlovsk region (Plotnikov \& Fefelov 2009); however, the study does not include descriptions and illustrations. The specimen is represented by a large colony of mature sporophores. The description of our specimen is consistent with the original description from Japan (Yamamoto 2000) and SEM-photographs of spores (Zhang \& Liu 2016). It was also found in China, Jilin Province (Zhang \& Liu 2016); Finland (Varis et al. 2016), Norway, and the Netherlands (GBIF.org).

* Stemonitis pallida Wingate, in Macbride., FC: 3: 1 (MYX 7142); 4: 1 (MYX 7157); 7: 3 (MYX 7221); 10: 1 (MYX 7245); 11: 1 (MYX 7255).

†Stemonitis splendens Rostaf., FC: 4: 1 (MYX 7160); 6: 1 (MYX 7200); 7: 1 (MYX 7210); 10: 1 (MYX 7267).

†Stemonitopsis aequalis (Peck) Y. Yamam., FC: 4: 1; 7: 1 (MYX 7211).

*Stemonitopsis gracilis (G. Lister) Nann.-Bremek., FC: 1: 1 (MYX 7136).

†Stemonitopsis hyperopta (Meyl.) Nann.-Bremek., FC: 7: 1 (MYX 7223); 10: 1 (MYX 7244); 25: 2 (MYX 7316); 26: 1.

†Stemonitopsis typhina (F.H. Wigg.) Nann.-Bremek., FC: 1: 1 (MYX 7133); 4: 3 (MYX 7159); 6: 2 (MYX 7197); 25: 3 (MYX 7318, MYX 7320); 41: 4 (MYX 6371, MYX 6374, MYX 6374, MYX 6374); 47: 2 (MYX 6366, MYX 6366); 52: 1.

Trichia botrytis (J.F. Gmel.) Pers., FC: 52: 1; L2.

Trichia decipiens (Pers.) T. Macbr., FC: 7: 1 (MYX 7217); 22: 3 (MYX 7286); L2.

Trichia favoginea (Batsch) Pers., FC: 7: 1 (MYX 7218); 10: 1 (MYX 7242); 13: 1 (MYX 7263); 19: 1 (MYX 7272); 20: 1 (MYX 7279); 25: 1; 28: 1; L1; L4.

*Trichia lutescens (Lister) Lister., FC: 51: 1.

†Trichia persimilis P. Karst., FC: 6: 1 (MYX 7195).

†Trichia scabra Rostaf., FC: 10: 3 (MYX 7240); 22: 1 (MYX 7284); 30: 1.

†Trichia subfusca Rex., FC: 50: 1; 52: 1.

Trichia varia (Pers. ex J.F. Gmel.) Pers., FC: L2.

Tubifera ferruginosa (Batsch) J.F. Gmel., FC: 4: 1 (MYX 7149); 6: 2 (MYX 7186); 7: 4 (MYX 7208); 10: 1 (MYX 7237); L2

\section{DISCUSSION}

In 2015-2016, 494 specimens were obtained for this study including 74 species and 6 varieties from 23 genera, 12 families, and 6 orders. Of these myxomycetes, 456 specimens ( 55 species) were collected in the field and 38 (23 species) were obtained from moist chamber cultures on substrates collected on Petrov Island. Only four species were simultaneously revealed by both of these methods. This confirms the need for their joint usage for comprehensive studies of Myxomycetes diversity. 
Fifty seven species had not been reported from Lazovsky Nature Reserve before this study, and 15 species were described as new for Primorye Territory. One species, Clastoderma pachypus, was recorded for the Russian Far East for the first time.

Substrates for the moist chambers cultures were collected only from Petrov Island, from Taxus cuspidata grove. For this reason, only a part of species was revealed, that can be obtained by this method from studied area.

The sampling effort for the field collections were probably sufficient to recover all of the most common species (55 taxa from 457 records, $81 \%$ complete according to the Chao 2 estimator). The large majority (44\%) of taxa are rare, representing $<0.5 \%$ of the total abundance (Stephenson et al. 1993). Only eight species are considered abundant, representing $>3 \%$ of the total abundance in field collections, including Hemitrichia calyculata (48 specimens/ $10.6 \%$, Stemonitis axifera (47/10.4\%), Ceratiomyxa fruticulosa (42/9.3\%), Lycogala epidendrum (40/8.8 \%), Comatricha nigra (35/7.7\%), Arcyria cinerea (32/6.9\%), Physarum viride (16/ $3.5 \%$ ), and Stemonitopsis typhina (16/3.5\%). Together these eight taxa represent $60 \%$ of the total abundance in field collections.

Consequently, taking into account the literature data (Novozhilov \& Krussanova 1988), 90 species from 27 genera, 12 families, and all 6 orders were found on the territory of Lazovsky Nature Reserve and its buffer zone. These records bring the total number of Myxomycetes species reported from Primorye Territory to 187 . This number indicates that currently, Primorye Territory (despite almost complete lack of regular studies, except for the SikhoteAlin Nature Reserve) is one of the regions with the greatest myxomycetes species diversity, inhabited by approximately $43.5 \%$ of all known species cited from Russia (Matveev et al. 2015-2019).

Only 16 previously found species (48\%) are recorded in this study again, three decades later. Such differences can be explained by the seasonality of the sporophore formation in these species: in 1986, studies were performed in late September - early October, while in 2015 and 2016, material was collected from mid June to mid August.

The majority of the species in our study belongs to the order Trichiales (26 species), Physarales (25), and Stemonitidales (21). Fewer species were recorded in the order Cribrariales (14). Only three species belong to Echinosteliales, and a single species to Ceratiomyxales. For well-studied territories, the proportion of the species of Physarales is slightly higher than that from all other orders (Novozhilov \& Fefelov 2001, Novozhilov et al. 2010, Vlasenko \& Novozhilov 2011a, Gmoshinskiy 2013). On one hand, this is due to the large number of described species in Physarales, and on the other hand, some members of this order often have a pronounced seasonality in the formation of sporophores (Vlasenko \& Novozhilov 2011b, Gmoshinskiy \& Matveev 2016) and relatively fragile fruit bodies, which quickly collapse. At the same time, sporophores of Trichiales do not contain lime and remain relatively long in the field conditions. Thus, to obtain an exhaustive species list, research should be conducted during the entire vegetation period, from the moment when snow melts to the time when it falls. In addition, it is preferable that such work is performed during several field seasons, since the species diversity varies from year to year even within the same study area (Barsukova et al. 2012).

\section{CONCLUDING REMARKS}

The myxomycetes biota of Lazovsky Nature Reserve currently includes 90 species. However, it should be recognized that the species diversity of these organisms is far from being fully studied. When planning further research, special attention should be paid to the core of the reserve and the survey of the mountainous portion of the reserve. In this case, the collection of material should be carried out not only during summer but the whole snowless period. In the course of further research, extensive use of the moist chamber method is also essential, for which the main types of plant substrates should be selected.

\section{ACKNOWLEDGEMENTS}

We are grateful to the administration of Lazovsky Nature Reserve for help in organization of fieldwork in 2015-2016. We thank E.A. Antonov and I.A. Viner for collected material. We are grateful to the members of Interdepartmental Electron Microscopy Laboratory (Faculty of Biology, Lomonosov MSU) for their technical support. Identification of the material used in the present study was supported by Russian Foundation for Basic Research (project no. 18-04-01232 A) and the state program of BIN RAS (no. AAAA-A19-119020890079-6). Work of the first author was supported by Moscow State University Grant for Leading Scientific Schools "Depository of the Living Systems" in frame of the MSU Development Program.

\section{LITERATURE CITED}

Adl, S.M., D. Bass, C.E. Lane, J. Lukeš, C.L. Schoch, A. Smirnov, S. Agatha, C. Berney, M.W. Brown, F. Burki ... Q. Zhang. 2019. Revisions to the classification, nomenclature, and diversity of Eukaryotes. Journal of Eukaryotic Microbiology 66(1):4-119.

Barsukova, T.N., V.I. Gmoshinskiy, V.P. Prokhorov \& E.A. Dunaev 2012. The slime moulds of S.N. Skadovsky Zvenigorod Biological station of Moscow State University. Mikologiya i fitopatologiya 46(2):111-121 (in Russian with English summary). [Барсукова Т.Н., Гмошинский В.И., Прохоров В.П., Аунаев Е.А. 2012. Миксомицеты Звенигородской биологической станции им. С.Н. СкаАовского Московского государственного университета им. М.В. Аомоносова // Микология и фитопатомогия. Т. 46, Вып. 2. С. 111-121].

Bunkina, I.A. \& M.M. Nazarova 1978. Fungi. In: Flora and vegitation of Ussuriysky reserve, pp. 36-39, Nauka, Moscow (in Russian) [Бункина И.А., Назарова М.М. 1978. Грибы / / ФАора и растительность Уссурийского заповеАника. Москва: Наука. С. 36-39].

Bunkina, I.A. \& E.Z. Koval' 1967. On flora of Myxomycetes in Primorye Territory. Novosti sistematiki nizshikh rasteniy 4: 151-152. (in Russian) [Бункина И.А., Коваль Э.3. 1967. К фморе миксомицетов Приморского края // Новости систематики низших растений. Т. 4. С. 151-152].

Egorova, L.N. (ed.) 2002. Flora, mycobiota and vegetation of the Lazousky reserve. Ostrov Russkiy, Vladivostok, 216 pp. (in 
Russian with English abstract). [ФАора, микобиота и растительность Аазовского заповедника / под реА. А.Н. Егоровой. 2002. ВАадивосток: Остров Русский. 216 с.].

GBIF.org Stemonitis marjana Y. Yamam. Checklist dataset. (accessed on 05.03.2019). https://doi. org/10.15468/39omei

Gmoshinskiy, V.I. 2013. Myxomycetes of Moscow and Moscow region ( $\mathrm{PhD}$ thesis). Moscow, 690 pp. (in Russian) [Tмошинский В.И. 2013. Миксомицеты Москвы и Московской области (Аиссертация на соискание уч. ст. к.б.н.). Москва: МГУ. 690 с.].

Gmoshinskiy V.I. \& E.A. Antonov 2016. On the Myxomycetes biota of Primorye Territory. Bulleten' Botanicheskogo Sada-Instituta DVO RAN 15:16-19 (in Russian with English abstract). [Гмошинский В.И., Антонов Е.А. К биоте миксомицетов Приморья // Бюлмютень Ботанического сада-института АВО РАН. Т. 15. С. 16-19].

Gmoshinskiy, V.I. \& A.V. Matveev 2016. Seasonal dynamics of sporophore formation in myxomycetes of Moscow and Moscow region. Mikologiya i Fitopatologiya 50(3):139-147.

Ing, B. 1999. The myxomycetes of Britain and Ireland. The Richmond Publishing Co. Ltd., London, 374 pp.

Kowalski, D.T. 1975. The myxomycete taxa described by Charles Meylan. Mycologia 67(3):448-494.

Lado, C. 1985. Estudios sobre Myxomycetes. VI. Anales Jardín botánico de Madrid 42(1):9-23.

Lado, C. 2005-2019. An on line nomenclatural information system of Eumycetozoa. Real Jardín Botánico, CSIC. Madrid, Spain. http://www.nomen.eumycetozoa.com. (accessed 16.09.2019).

Lado, C. \& F. Pando 1997. Myxomycetes. I. Ceratiomyxales, Echinosteliales, Liceales, Trichiales. Flora Mycologica Iberica. 2:1-323 pp.

Lado, C. \& U. Eliasson 2017. Taxonomy and systematics: current knowledge and approaches on the taxonomic treatment of Myxomycetes. In: Myxomycetes: biology, systematics, biogeography and ecology, 1st edition (S.L. Stephenson, C.A. Rojas, eds), pp. 205-251, Academic Press, London.

Leontyev, D.V., R. McHugh, K.A. Fefelov \& A.V. Kochergina 2011. New and rare Myxomycetes of Ukraine. 2. Southwest Crimea. Nova Hedwigia 92 (1-2):245-256.

Liu, Q.S., S.Z. Yan, J.Y. Dai \& S.L. Chen 2013. Species diversity of corticolous myxomycetes in Tianmu Mountain National Nature Reserve, China. Canadian Journal of Microbiology 59(12):803-813.

Matveev, A.V., F.M. Bortnikov, V.I. Gmoshinsky \& Yu.K. Novozhilov 2016-2019. Myxomycetes of Russia. Web application. http://myxomycetes.org/russia (accessed 17.09.2019).

Mitchell, D.W. 1978. A key to the corticolous myxomycetes. Part. 1. Bulletin of the British Mycological Society 12(1):18-60.

Nannenga-Bremekamp, N.E. 1968. Notes on myxomycetes XV. New species of Oligonema, Licea, Clastoderma, Comatricha, Paradiacheopsis and Badhamia. Proceedings van de Koninklijke Nederlandse Akademie van Wetenschappen Section C 71(1):41-51.

Novozhilov, Yu.K. \& Z.G. Krusanova 1989. Myxomycetes from Lazovski reserve (Primorye Territory). Novosti Sistematiki Nizshikh Rasteniy 26:69-72 (in Russian). [Новожилов Ю.К., Крусанова 3.Г. 1989. Миксомицеты Аазовского заповедника (Приморский край) // Новости систематики низших растений. Т. 26. С. 69-72].
Novozhilov, Yu.K. \& K.A. Fefelov 2001. An annotated checklist of the myxomycetes of Sverdlovsk region, West Siberian lowland, Russia. Mikologiya i Fitopatologiya 35(4): 41-52.

Novozhilov, Yu.K., M. Schnittler, A.V. Vlasenko \& K.A. Fefelov 2010. Myxomycete diversity of the Altay Mountains (southwestern Siberia, Russia). Mycotaxon 111(1):91-94.

Novozhilov, Yu.K., A.W. Rollins \& M. Schnittler 2017a. Ecology and distribution of Myxomycetes. In: Myxomycetes: biology, systematics, biogeography and ecology, 1st ed. (S.L. Stephenson, C.A. Rojas, eds), pp. 253-297, Academic Press, London.

Novozhilov, Yu.K., M. Schnittler, D.A. Erastova \& O.N. Shchepin 2017b. Myxomycetes of the Sikhote-Alin State Nature Biosphere Reserve (Far East, Russia). Nova Hedwigia 104(1-3):183-209.

Plotnikov, B.S. \& K.A. Fefelov 2009. Myxomycetes under the pollution gradient of a cooper plant in southern taiga, the middle Urals. Mikologiva i Fitopatologiya 43(1):33-44 (in Russian with English abstract). [П Аотников Б.С., Фефелов К.А. 2009. Миксомицеты южной тайги среднего Урала в градиенте промышленного загрязнения выбросами медеплавильного комбината // Микология и фитопатология. Т. 43, вып. 1. С. 33-44].

Poulain M., M. Meyer \& J. Bozonnet 2011a. Les Myxomycétes. Tome 1. Guide de détermination. Fédération mycologique et botanique Dauphiné-Savoie. Sévrier, France, 568 pp.

Poulain, M., M. Meyer \& J. Bozonnet 2011b. Les Myxomycétes. Tome 2. Planches. Fédération mycologique et botanique Dauphiné-Savoie. Sévrier, France, 544 pp.

Schnittler, M., Yu.K., Novozhilov, E. Carvajal \& F.W. Spiegel 2013. Myxomycete diversity in the Tarim basin and eastern Tian-Shan, Xinjiang Prov., China. Fungal Diversity 59(1):91-108.

Stephenson, S.L. 1989. Distribution and ecology of myxomycetes in temperate forests. II. Patterns of occurrence on bark surface of living trees, leaf litter, and dung. Mycologia 81(4):608-621.

Stephenson, S.L., I. Kalyanasundaram \& T.N. Lakhanpal 1993. A comparative biogeographical study of myxomycetes in the mid-Appalachians of eastern North America and two regions of India. Journal of Biogeography 20(6):645-657.

Stephenson, S.L. \& H. Stempen 1994. Myxomycetes: A handbook of slime molds. Timber Press, Portland, Oregon, $183 \mathrm{pp}$.

Varis, E., A. Karhilahti \& A. Prättälä 2016. Eleven Myxomycete species new to Finland. Karstenia 56(1-2):61-72.

Vasilyev, N.G., E.N. Matyushin \& E.V. Kuptsov 1985. Lazovsky reserve of L.G. Kaplanov. In: Reserves of USSR. Reserves of Far East (V.E. Sokolov \& E.E. Syroechkovskiy, eds), pp. 225-249, Mysl', Moscow (in Russian). [Васильев Н.Г., Матюшин Е.Н., Купцов Е.В. Аазовский заповеАник им. А.Г. Капланова // Заповедники СССР. Заповедники Аальнего Востока / под ред. В.Е. Соколова, Е.Е. Сыроечковского. М.: Мысль. С. 225-249].

Vlasenko, A.V. \& Yu.K. Novozhilov 2011a. Taxonomical structure of myxomycetes biota in the pine forests on the right-bank part the Upper Ob' river. Mikologiva $i$ Fitopatologiya 45(6):478-488 (in Russian with English abstract). [ВАасенко А.В., Новожилов Ю.К. 2011. Таксономическая структура биоты миксомицетов сосновых месов правобережной части верхнего Приобья // Микология и фитопатология. Т. 45, вып. 6. С. 478-488].

Vlasenko, A.V. \& Yu.K. Novozhilov 2011b. Phenological features of Myxomycetes in the pine forests on the 
right-bank part of the Upper Ob' river. Rastitel'nyi mir Aziatskoi Rossii (2):3-8 (in Russian with English abstract). [Вıасенко А.В., Новожилов Ю.К. 2011а. Фенологические особенности миксомицетов сосновых месов правобережной части верхнего Приобья // Растительный мир Азиатской России. № 2(8). С. 3-8].
Yamamoto, Y. 2000. A new species of Stemonitis (Myxomycetes) from Japan. Karstenia 40:197-198.

Zhang, B. \& L. Yu 2016. A new species, Stemonitis sichuanensis, and a newly recorded species, Stemonitis marjana (Myxogastria) from China. Phytotaxa 258(2):195-199. 